\title{
ON THE DISTRIBUTION OF FIRST HITS FOR THE SYMMETRIC STABLE PROCESSES
}

\author{
BY
}

R. M. BLUMENTHAL, R. K. GETOOR AND D. B. RAY(1)

1. Introduction. Let $\{X(t) ; t \geqq 0\}$ be the symmetric stable process in $R^{N}$ of index $\alpha$ with $0<\alpha \leqq 2$; that is, a process with stationary independent increments whose continuous transition density, relative to Lebesgue measure in $R^{N}$, is

$$
p(t, x)=(2 \pi)^{-N} \int e^{i(x, \xi)} e^{-t|\xi|^{\alpha}} d \xi .
$$

Here $t>0, x$ and $\xi$ are points in $R^{N}, d \xi$ is $N$-dimensional Lebesgue measure, $(x, \xi)$ is the usual inner product in $R^{N}$, and $|\xi|^{2}=(\xi$, $\xi$ ). Throughout this paper integrals will be over all of $R^{N}$ unless explicitly stated otherwise. Of course, to determine our process we must also specify the distribution of $X(0)$. We will always assume that our process starts from some fixed point $x$ in $R^{N}$; that is, $X(0)=x$ with probability one. We will write $P_{x}$ and $E_{x}$ for probabilities and expectations under the condition $X(0)=x$. We will assume that the sample functions are normalized to be right continuous and to have left limits everywhere. See [2, §2] for a complete description of this setup.

Define

$$
\begin{aligned}
T & =\inf \{t:|X(t)|>1\}, \\
T^{*} & =\inf \{t:|X(t)|<1\} .
\end{aligned}
$$

It is easy to see that $T$ and $T^{*}$ are random variables, $T$ being the first passage time to the exterior of the unit ball and $T^{*}$ the first passage time to the interior of the unit ball. Let

$$
\begin{aligned}
\mu_{x}(d y) & =P_{x}[X(T) \in d y, T<\infty], \\
\mu_{x}^{*}(d y) & =P_{x}\left[X\left(T^{*}\right) \in d y, T^{*}<\infty\right] .
\end{aligned}
$$

Thus $\mu_{x}$ and $\mu_{x}^{*}$ are, respectively, the distributions of first hits of the exterior and interior of the unit ball when $X(0)=x$. Of course, $\mu_{x}(\{y:|y|<1\})=0$ and $\mu_{x}^{*}(\{y:|y|>1\})=0$.

Define

Received by the editors December 19, 1960.

(1) The research of the first two authors was supported, in part, by the National Science Foundation, and that of the last, by the Alfred P. Sloan Foundation. 
(1.4) $f(x ; y)=\left.\pi^{-(N / 2+1)} \Gamma\left(\frac{N}{2}\right) \sin \frac{\pi \alpha}{2}|1-| x\right|^{2}|\alpha / 2| 1-\left.|y|^{2}\right|^{-\alpha / 2}|x-y|^{-N}$.

The main results of this paper are the following theorems:

Theorem A. If $0<\alpha<2$ and $|x|<1$, then

$$
\mu_{x}(d y)=f(x ; y) d y, \quad|y| \geqq 1 .
$$

TheOREM B. If $\alpha<N$ or if $\alpha=N=1$, then for $|x|>1$,

$$
\mu_{x}^{*}(d y)=f(x ; y) d y,
$$

Theorem C. If $N=1<\alpha<2$ and $|x|>1$, then

$$
\begin{array}{r}
\mu_{x}^{*}(d y)=f(x ; y) d y-(\alpha-1) \pi^{-1} \sin \frac{\pi \alpha}{2} \int_{1}^{|x|}\left(u^{2}-1\right)^{\alpha / 2-1} d u\left(1-y^{2}\right)^{-\alpha / 2} d y, \\
|y| \leqq 1 .
\end{array}
$$

Theorem A was obtained by Spitzer [9] in the case $N=1$ and $\alpha=1$, and by Widom [10] in the case $N=1$ and $0<\alpha<2$. We are informed the result has also been proved by Kesten and J. R. Kinney, independently.

Relatively straightforward computations yield the following corollaries:

Corollary 1. Let $N=1$ and for $|x|<1$ define $p(x)=P_{x}[X(T) \geqq 1, T<\infty]$ : that is, the probability that the process starting from $x$ first leaves the interval $[-1,1]$ to the right. Then

$$
p(x)=2^{1-\alpha} \Gamma(\alpha)\left[\Gamma\left(\frac{\alpha}{2}\right)\right]^{-2} \int_{-1}^{x}\left(1-u^{2}\right)^{\alpha / 2-1} d u .
$$

Again in the case $\alpha=1$ this is due to Spitzer [9].

Corollary 2. For $\alpha<N$ and $|x|>1$, define $q(x)$ to be the probability that the process starting from $x$ never hits the unit ball: $q(x)=P_{x}\left[T^{*}=\infty\right]$. Then

$$
q(x)=\Gamma\left(\frac{N}{2}\right)\left[\Gamma\left(\frac{N-\alpha}{2}\right) \Gamma\left(\frac{\alpha}{2}\right)\right]^{-1} \int_{0}^{|x|^{2}-1}(u+1)^{-N / 2} u^{\alpha / 2-1} d u
$$

Corollary 3. Let $N=1$ and $1 \leqq \alpha<2$. For $|x|>1$ and $t>0$, let $r(x, t)$ be the probability that the process starting from $x$ has not hit the unit ball by time $t: r(x, t)=P_{x}\left[T^{*}>t\right]$. Then

$$
\lim _{t \rightarrow \infty} \log \operatorname{tr}(x, t)=\log \left(|x|+\left(x^{2}-1\right)^{1 / 2}\right), \quad \text { if } \alpha=1,
$$




$$
\begin{aligned}
\lim _{t \rightarrow \infty} t^{1-1 / \alpha} r(x, t)=\alpha \sin \frac{\pi \alpha}{2} \Gamma(2-\alpha) & {\left[\Gamma\left(1-\frac{1}{\alpha}\right)\right]^{-1}\left[\Gamma\left(\frac{1}{\alpha}\right)\right]^{-2} } \\
\cdot & \int_{1}^{|x|}\left(u^{2}-1\right)^{\alpha / 2-1} d u, \quad \text { if } 1<\alpha<2 .
\end{aligned}
$$

The last result is a corollary of the technique of the proof rather than of Theorems $\mathrm{A}, \mathrm{B}$, and $\mathrm{C}$ themselves. The distinction between the cases $0<\alpha<1$ and $1 \leqq \alpha<2$ when $N=1$ reflects the fact that the one-dimensional symmetric stable process is transient if $0<\alpha<1$ and recurrent if $1 \leqq \alpha<2$.

Another consequence of Theorem A deserves mention. Let $F$ be a measurable subset of the unit ball. For $|x|<1$, let $H(x, F)$ be the expected amount of time the process starting at $x$ spends in $F$ before leaving the unit ball: that is,

$$
H(x, F)=E_{x}\left[\int_{0}^{T} V_{F}(X(t)) d t\right]
$$

where $V_{F}$ is the characteristic function of $F$. For each $x,|x|<1, H(x, \cdot)$ is a measure on the unit ball.

COROllary 4. For $|x|<1$ and $|y| \leqq 1$,

$$
H(x, d y)=2^{-\alpha} \pi^{-N / 2}\left[\Gamma\left(\frac{\alpha}{2}\right)\right]^{-2} \Gamma\left(\frac{N}{2}\right) \int_{0}^{z}(u+1)^{-N / 2} u^{\alpha / 2-1} d u|x-y|^{\alpha-N} d y,
$$

where $z=\left(1-|x|^{2}\right)\left(1-|y|^{2}\right)|x-y|^{-2}$.

Corollary 4 was obtained by $\mathrm{Kac}$ and Pollard [3] in the case $N=\alpha=1$; by Kesten [5] in the case $N=1,0<\alpha<1$; and by Widom [10] in the case $N=1,0<\alpha<2$, in a different form.

In [10], Widom uses techniques not unrelated to those of this paper. Spitzer [9] used the special relation of the Cauchy process $(\alpha=N=1)$ to the two-dimensional Brownian motion; and $\mathrm{Kac}$ and Pollard [3] used the special behavior of the Tchebycheff polynomials under the generator of the Cauchy process. In [5], Kesten applies the result we call Theorem A here to obtain some interesting and deep limit theorems for Toeplitz matrices.

The techniques of this paper consist, in the case $\alpha<N$, simply of noticing that $\mu_{x}$ is the harmonic measure of the unit ball corresponding to the Riesz potential of exponent $\alpha$ in $R^{N}$. As such it was calculated by Riesz [7] in 1938. The extensions necessary to cover the case $N=1 \leqq \alpha<2$ are due, in this context, to Kac [4]. Our results are stated in terms of the unit sphere, but they carry over immediately to an arbitrary sphere, since the symmetric stable processes are homogeneous in space and satisfy the scaling relationship 


$$
P_{x}[r X(t) \in B]=P_{r x}\left[X\left(r^{\alpha} t\right) \in B\right],
$$

which holds for all $r>0$ and all Borel sets $B$ in $R^{N}$.

2. Equations for the hitting probabilities. All the information necessary to determine the hitting probabilities $\mu_{x}$ and $\mu_{x}^{*}$ is contained in the "first passage time relation" or "Désiré-André equation" (cf. [6]). Let $A$ be an open set in $R^{N}, \bar{A}$ its closure. Define the random variable $\tau$ for a symmetric stable process starting from $x$ in $R^{N}$ by

$$
\tau=\inf \{t: X(t) \in A\} .
$$

For $B$ a measurable subset of $R^{N}$ and $s \geqq 0$, define

$$
\begin{aligned}
& H_{\bullet}(x, B)=E_{x}\left[\int_{0}^{\tau} e^{-s t} V_{B}[X(t)] d t\right], \\
& L_{s}(x, B)=E_{x}\left[e^{-s \tau} ; X(\tau) \in B, \tau<\infty\right] .
\end{aligned}
$$

Again, $V_{B}$ is the characteristic function of the set $B$. Finally, set

$$
R_{s}(y)=\int_{0}^{\infty} e^{-s t} p(t, y) d t
$$

where $p(t, y)$ is the transition density of the process, defined in (1.1). Then the first passage time relation, easily derived from the strong Markov property [1], states

$$
\int_{B} R_{s}(x-y) d y=H_{s}(x, B)+\int_{\bar{A}} L_{s}(x, d u) \int_{B} R_{s}(u-y) d y, \quad s>0 .
$$

From this it follows that $H_{s}(x, B)$ has a continuous density $H_{s}(x, y)$, satisfying

$$
R_{s}(x-y)=H_{s}(x, y)+\int_{\bar{A}} L_{s}(x, d u) R_{s}(u-y), \quad s>0 .
$$

Note that (2.1) is trivial if $x$ is in $A$, while if $y$ is in $A$ and $x$ is not, $H_{8}(x, y)=0$.

Now suppose that $\alpha<N$. Then one can calculate without much difficulty that

$$
\lim _{s \rightarrow 0} R_{\imath}(x)=\Gamma\left(\frac{N-\alpha}{2}\right)\left[2^{\alpha} \pi^{N / 2} \Gamma\left(\frac{\alpha}{2}\right)\right]^{-1}|x|^{\alpha-N}
$$

Since all the functions involved in (2.1) increase as $s$ decreases to zero, we obtain in the limit

$$
|x-y|^{\alpha-N}=\int_{\bar{A}} L_{0}(x, d u)|u-y|^{\alpha-N}, \quad x \notin A, y \in A,
$$




$$
\begin{aligned}
H_{\mathrm{n}}(x, y)=\Gamma\left(\frac{N-\alpha}{2}\right)\left[2^{\alpha} \pi^{N / 2} \Gamma\left(\frac{\alpha}{2}\right)\right]^{-1} \\
\cdot\left\{|x-y|^{\alpha-N}-\int_{\bar{A}} L_{0}(x, d u)|u-y|^{\alpha-N}\right\}, \quad x, y \notin A .
\end{aligned}
$$

These equations may be specialized further by making $A$ the unit ball or its exterior. Then $L_{0}(x, d u)=\mu_{x}^{*}(d u)$ or $\mu_{x}(d u)$; and if $A$ is the exterior of the unit ball, $H_{0}$ is the kernel $H$ defined in (1.5).

$$
\begin{array}{rlrl}
|x-y|^{\alpha-N} & =\int_{|u| \geq 1}|u-y|^{\alpha-N} \mu_{x}(d u), & |x|<1<|y| \\
|x-y|^{\alpha-N} & =\int_{|u| \leq 1}|u-y|^{\alpha-N} \mu_{x}^{*}(d u), & |y|<1<|x| \\
H(x, y) & =\Gamma\left(\frac{N-\alpha}{2}\right)\left[2^{\alpha} \pi^{N / 2} \Gamma\left(\frac{\alpha}{2}\right)\right]^{-1} & \\
\cdot\left\{|x-y|^{\alpha-N}-\int_{|u| \geq 1}|u-y|^{\left.\alpha-N_{\mu_{x}}(d u)\right\},}\right. & |x|,|y|<1 .
\end{array}
$$

If $N=1 \leqq \alpha<2, R_{s}(x)$ becomes infinite as $s$ tends to zero, but a technique which was used by $\mathrm{Kac}[4]$ on a similar problem may be applied. One has

$$
\lim _{s \rightarrow 0}\left[C_{\alpha}(s)-R_{s}(x)\right]=G_{\alpha}(x)
$$

where

$$
\begin{array}{lr}
C_{\alpha}(s)=\left[\alpha \sin \frac{\pi}{\alpha}\right]^{-1} s^{1 / \alpha-1}, & 1<\alpha<2, \\
C_{\alpha}(s)=-\frac{1}{\pi} \log s, & \alpha=1, \\
G_{\alpha}(x)=\sin \frac{\pi \alpha}{2} \Gamma(2-\alpha)[\pi(\alpha-1)]^{-1}|x|^{\alpha-1}, & 1<\alpha<2, \\
G_{\alpha}(x)=\frac{1}{\pi} \log |x|, & \alpha=1 .
\end{array}
$$

In fact, the function $C_{\alpha}(s)-R_{s}(x)$ increases to $G_{\alpha}(x)$ as $s$ decreases to zero, for each pertinent value of $\alpha$. Subtracting each side of (2.1) from $C_{\alpha}(s)$,

$$
\begin{aligned}
C_{\alpha}(s) & -R_{s}(x-y) \\
& =-H_{s}(x, y)+\int_{\bar{A}} L_{s}(x, d u)\left[C_{\alpha}(s)-R_{s}(u-y)\right]+C_{\alpha}(s)\left[1-L_{s}(x, \bar{A})\right] .
\end{aligned}
$$


In the limit,

$$
G_{\alpha}(x-y)=-H_{0}(x, y)+\int_{\bar{A}} L_{0}(x, d u) G_{\alpha}(u-y)+g_{\alpha}(x),
$$

where

$$
\begin{aligned}
g_{\alpha}(x) & =\lim _{s \rightarrow 0} C_{\alpha}(s)\left(1-L_{s}(x, \bar{A})\right) \\
& =\lim _{s \rightarrow 0} C_{\alpha}(s) E_{x}\left[1-e^{-s r}\right]
\end{aligned}
$$

exists when $y$ is in $A$ and $x$ is not, since all the other terms involved, being increasing and bounded, have limits. It is not hard to see that if the complement of $A$ is compact, then $E_{x}[\tau]<\infty$, so that $g_{\alpha}(x)=0$ and $H_{s}(x, y)$ is bounded, for each $x$ not in $A$.

We specialize again, in several directions, separating the cases

$$
\begin{gathered}
x \in A, y \in A ; x \in A, y \notin A ; \alpha=1 ; 1<\alpha<2 ; \\
A=(-1 ; 1) ; \quad A=(-\infty, 1) \cup(1, \infty) ;
\end{gathered}
$$

and their combinations: If $\alpha=1$,

$$
\begin{array}{ll}
\log |x-y|=\int_{|u| \geq 1} \log |u-y| \mu_{x}(d u), & |x|<1<|y| \\
\log |x-y|=\int_{|u| \leq 1} \log |u-y| \mu_{x}^{*}(d u)+k_{1}(x), & |y|<1<|x|
\end{array}
$$

where

$$
\begin{aligned}
k_{1}(x) & =\lim _{s \rightarrow 0}-\log s E_{x}\left[1-e^{-s T^{*}}\right] \\
H(x, y) & =-\frac{1}{\pi} \log |x-y|+\frac{1}{\pi} \int_{|u| \geq 1} \log |u-y| \mu_{x}(d u,)
\end{aligned}
$$$$
|x|,|y|<1 \text {. }
$$

If $1<\alpha<2$,

$$
\begin{array}{ll}
|x-y|^{\alpha-1}=\int_{|u| \geq 1}|u-y|^{\alpha-1} \mu_{x}(d u), & |x|<1<|y| \\
|x-y|^{\alpha-1}=\int_{|u| \leq 1}|u-y|^{\alpha-1} \mu_{x}^{*}(d u)+k_{\alpha}(x), & |y|<1<|x|,
\end{array}
$$

where 


$$
\begin{aligned}
k_{\alpha}(x)= & \pi(\alpha-1)\left[\alpha \sin \frac{\pi}{\alpha} \sin \frac{\pi \alpha}{2} \Gamma(2-\alpha)\right]^{-1} \lim _{s \rightarrow 0} s^{1 / \alpha-1} E_{x}\left[1-e^{-s T^{*}}\right], \\
H(x, y)= & \sin \frac{\pi \alpha}{2} \Gamma(2-\alpha)[\pi(\alpha-1)]^{-1} \\
& \cdot\left\{\int_{|u| \geq 1}|u-y|^{\alpha-1} \mu_{x}(d u)-|x-y|^{\alpha-1}\right\},|x|,|y|<1 .
\end{aligned}
$$

It will be seen that $(2.6)$ or $(2.10)$, together with the property $\mu_{x}^{*}([-1,1])$ $=1$, determine both $\mu_{x}^{*}$ and $k_{\alpha}$.

3. Some calculations of $M$. Riesz and extensions of them. Equations (2.2) and (2.3) state that $\mu_{x}$ and $\mu_{x}^{*}$ are the harmonic measures of the exterior and interior of the unit ball, corresponding to the Riesz potential of exponent $\alpha$ in $R^{N}$. Riesz has shown ([7, pp. 13-17] and [8]) that the unique solutions of (2.2) and (2.3) are those indicated in Theorems A and B. We repeat his calculations here, since they must be extended a bit to cover (2.4) and (2.5)(2.12).

The basis of the calculations in [7] is the idea of inversion in a sphere $\{u:|u-x|=r\}$ in $R^{N}$ : that is, the change of coordinates

$$
u \rightarrow v=x+r^{2}|u-x|^{-2}(u-x) \text {. }
$$

Riesz noted that if $f(u)$ is a potential of exponent $\alpha$ in $R^{N}$, then after inversion in a sphere with center $x,|x-v|^{\alpha-N} f(v)$ is a potential. This enabled him to calculate the harmonic measure of a ball from its equilibrium distribution, as we will do in this section. Besides the equilibrium distribution, we shall also evaluate the equilibrium potential outside the ball.

To avoid repetition of lengthy formulas, let us set, recalling the definition of $f(x ; y)$ in $(1.4)$,

$$
\begin{aligned}
g(y) & =\left.\left.|1-| x\right|^{2}\right|^{-\alpha / 2}|x-y|^{N} f(x ; y) \\
& =\left.\left.\pi^{-(N / 2+1)} \sin \frac{\pi \alpha}{2} \Gamma\left(\frac{N}{2}\right)|1-| y\right|^{2}\right|^{-\alpha / 2} \\
h(x ; y) & =\Gamma\left(\frac{N}{2}\right)\left[\Gamma\left(\frac{\alpha}{2}\right) \Gamma\left(\frac{N-\alpha}{2}\right)\right]^{-1} \int_{0}^{z}(r+1)^{-N / 2} r^{\alpha / 2-1} d r
\end{aligned}
$$

where

$$
\begin{aligned}
& z=\left.|1-| x\right|^{2}|| 1-|y|^{2}|| x-\left.y\right|^{-2}, \\
& h(y)=\lim _{|x| \rightarrow \infty} h(x, y) \\
&=\Gamma\left(\frac{N}{2}\right)\left[\Gamma\left(\frac{\alpha}{2}\right) \Gamma\left(\frac{N-\alpha}{2}\right)\right]^{-1} \int_{0}^{|y|^{2}-1}(r+1)^{-N / 2} r^{\alpha / 2-1} d r, \\
& \quad|y|>1 .
\end{aligned}
$$


Lemma 3.1. If $0<\alpha<2$, then

$$
\begin{aligned}
\int_{|u| \leq 1}|u-y|^{\alpha-N} g(u) d u & =1, & & |y| \leqq 1 \\
& =1-h(y), & & |y| \geqq 1 .
\end{aligned}
$$

Proof. If $|y|>1$, inversion in the sphere $\left\{u:|y-u|^{2}=|y|^{2}-1\right\}$ yields

$$
\begin{aligned}
\int_{|u| \leq 1}\left(1-|u|^{2}\right)^{-\alpha / 2}|u-y|^{\alpha-N} d u & \\
& =\left(|y|^{2}-1\right)^{\alpha / 2} \int_{|v| \leq 1}\left(1-|v|^{2}\right)^{-\alpha / 2}|v-y|^{-N} d v .
\end{aligned}
$$

Set $\omega_{n}=2 \pi^{n / 2}[\Gamma(n / 2)]^{-1}$, if $n \geqq 1$; then when $N \geqq 2$,

$$
\begin{aligned}
\int_{|v| \leq 1} & \left(1-|v|^{2}\right)^{-\alpha / 2}|v-y|^{-N} d v \\
= & \omega_{N-1} \int_{0}^{1} d r r^{N-1}\left(1-r^{2}\right)^{-\alpha / 2} \int_{0}^{\pi} d \theta \sin ^{N-2} \theta\left(r^{2}+|y|^{2}-2 r|y| \cos \theta\right)^{-N / 2} \\
= & \omega_{N}|y|^{2-N} \int_{0}^{1} d r r^{N-1}\left(1-r^{2}\right)^{-\alpha / 2}\left(|y|^{2}-r^{2}\right)^{-1}
\end{aligned}
$$

by the Poisson formula in $R^{N}$. The end result is trivially the same if $N=1$.

We shall complete the calculation only for the case $\alpha<N$; the changes necessary when $N=1$ and $1<\alpha<2$ are fairly obvious.

$$
\begin{aligned}
\Gamma\left(\frac{\alpha}{2}\right) \Gamma\left(\frac{N-\alpha}{2}\right)\left[\Gamma\left(\frac{N}{2}\right)\right]^{-1} \int_{0}^{1} d r r^{N-1}\left(1-r^{2}\right)^{-\alpha / 2}\left(|y|^{2}-r^{2}\right)^{-1} \\
\quad=2 \int_{0}^{1} d r r\left(1-r^{2}\right)^{-\alpha / 2}\left(|y|^{2}-r^{2}\right)^{-1} \int_{0}^{r} t^{N-\alpha-1}\left(r^{2}-t^{2}\right)^{(\alpha-2) / 2} d t \\
\quad=\int_{0}^{1} d t t^{N-\alpha-1} \int_{0}^{1-t^{2}} d s s^{-\alpha / 2}\left(1-t^{2}-s\right)^{\alpha / 2-1}\left(s+|y|^{2}-1\right)^{-1} \\
=\left(|y|^{2}-1\right)^{-\alpha / 2} \pi \csc \frac{\pi \alpha}{2} \int_{0}^{1} d t t^{N-\alpha-1}\left(|y|^{2}-t^{2}\right)^{\alpha / 2-1} \\
=\left(|y|^{2}-1\right)^{-\alpha / 2}|y|^{N-2} \frac{\pi}{2} \csc \frac{\pi \alpha}{2} \int_{|y| 2-1}^{\infty} d r(r+1)^{-N / 2} r^{\alpha / 2-1}
\end{aligned}
$$

Combining the three sets of equations gives (3.5) when $\alpha<N$.

If $|y|<1$ invert in the sphere $\left\{u:|u-y|^{2}=1-|y|^{2}\right\}$ and reflect in the point $y$ : the combined change of coordinates is the same as before: $u \rightarrow v$ $=y-\left(1-|y|^{2}\right)|u-y|^{-2}(u-y)$. Then proceeding as before, 


$$
\begin{aligned}
\int_{|u| \leq 1} & \left(1-|u|^{2}\right)^{-\alpha / 2}|u-y|^{\alpha-N} d u \\
= & \int_{|v| \geq 1}\left(1-|y|^{2}\right)^{\alpha / 2}\left(|v|^{2}-1\right)^{-\alpha / 2}|v-y|^{-N} d v \\
= & \omega_{N}\left(1-|y|^{2}\right)^{\alpha / 2} \int_{1}^{\infty} d r r\left(r^{2}-1\right)^{-\alpha / 2}\left(r^{2}-|y|^{2}\right)^{-1} \\
= & \frac{1}{2} \omega_{N} \pi \csc \frac{\pi \alpha}{2},
\end{aligned}
$$

the required result.

LEMMA 3.2. If $0<\alpha<2$ and $|x|>1$, then

$$
\int_{|u| \leq 1} f(x ; u)|y-u|^{\alpha-N} d u=|x-y|^{\alpha-N}, \quad|y| \leqq 1
$$

If $|x|<1$, then

$$
\begin{array}{rlrl}
\int_{|u| \geq 1} f(x ; u)|y-u|^{\alpha-N} d u & =|x-y|^{\alpha-N}, & & |y| \geqq 1, \\
& =|x-y|^{\alpha-N}(1-h(x ; y)), & |y| \leqq 1 .
\end{array}
$$

Proof. Equation (3.6) is obtained from (3.4) by inversion in the sphere $\left\{u:|u-x|^{2}=|x|^{2}-1\right\} ;$ (3.7) then follows by inversion in the unit sphere about the origin. These two inversions applied to (3.5) yield (3.8).

Lemma 3.3. Let $N=\alpha=1$. Then

$$
\int_{|u| \leq 1} g(u) \log |y-u| d u=-\log 2, \quad|y| \leqq 1 .
$$

If $|x|>1$, then

$$
\begin{aligned}
& \int_{|u| \leq 1} f(x ; u) \log |y-u| d u \\
& =\log |y-x|-\log \left[|x|+\left(x^{2}-1\right)^{1 / 2}\right], \quad|y| \leqq 1 .
\end{aligned}
$$

If $|x|<1$, then

$$
\begin{gathered}
\int_{|u| \geqq 1} f(x ; u) \log |y-u| d u=\log |y-x|, \quad|y| \geqq 1, \\
=\log \left[1-x y+\left(1-x^{2}\right)^{1 / 2}\left(1-y^{2}\right)^{1 / 2}\right], \quad|y| \leqq 1,
\end{gathered}
$$


Proof. It is a standard result, of course, that

$$
\begin{aligned}
\int_{|u| \leq 1} g(u) \log |y-u| d u & =-\log 2, & & |y| \leqq 1, \\
& =\log \left[|y|+\left(y^{2}-1\right)^{1 / 2}\right]-\log 2, & & |y| \geqq 1 .
\end{aligned}
$$

We can reduce the rest of Lemma 3.3 to this by the same changes of coordinates as before.

Let $w=(y x-1)(y-x)^{-1}$. Making the substitution $v=(u x-1)(u-x)^{-1}$,

$$
\begin{aligned}
& \pi \int_{|u| \leq 1} f(x ; u) \log |y-u| d u \\
& =\left(x^{2}-1\right)^{1 / 2} \int_{|u| \leq 1}\left(1-u^{2}\right)^{-1 / 2}|x-u|^{-1} \log |y-u| d u \\
& \quad=\int_{|v| \leq 1}\left(1-v^{2}\right)^{-1 / 2} \log \left[|w-v||y-x||x-v|^{-1}\right] d v \\
& =\pi \log |y-x|-\pi \log \left[|x|+\left(x^{2}-1\right)^{1 / 2}\right]
\end{aligned}
$$

if $|x|>1>|y|$. And if $|x|<1$, the same substitution yields

$$
\begin{aligned}
& \pi \int_{|u| \geq 1} f(x ; u) \log |y-u| d u \\
& =\int_{|v| \leq 1}\left(1-v^{2}\right)^{-1 / 2} \log \left[|w-v||y-x||x-v|^{-1}\right] d v \\
& =\pi \log |y-x| \text {, } \\
& =\pi \log \left\{|y-x|\left[|w|+\left(w^{2}-1\right)^{1 / 2}\right]\right\} \\
& =\pi \log \left[1-x y+\left(1-x^{2}\right)^{1 / 2}\left(1-y^{2}\right)^{1 / 2}\right] \text {, } \\
& |y|<1
\end{aligned}
$$

since $|w|<1$ if and only if $|y|>1$.

4. Uniqueness of the solutions of the equations. That (2.2) and (2.3) uniquely determine the hitting probabilities $\mu_{x}$ and $\mu_{x}^{*}$ when $\alpha<N$ is a standard result in potential theory. Again, we owe to $\mathrm{Kac}$ the extension of the argument necessary to treat (2.5), (2.6), (2.9) and (2.10).

Since the left side of (2.2) is bounded for $|y| \geqq 1$, when $x$ is a fixed point inside the unit ball, the energy integral

$$
\int_{|u| \geq 1} \int_{|y| \geq 1}|u-y|^{\alpha-N} \mu_{x}(d u) \mu_{x}(d y)
$$

is finite. Similarly $\mu_{x}^{*}$ has finite energy when $|x|>1$. Hence the uniqueness for (2.2) and (2.3) follows from the following well-known result. 
Lemma 4.1. Let $A$ be a closed subset of $R^{N}$, and let $0<\alpha<2, \alpha<N$. Let $\nu$ be a bounded signed measure on $A$ whose total variation $|\nu|$ has finite energy:

$$
\int_{A}|\nu|(d u) \int_{A}|\nu|(d y)|u-y|^{\alpha-N}<\infty .
$$

Then

$$
\int_{A}|u-y|^{\alpha-N} \nu(d u)=0, \quad y \in A
$$

implies $\nu=0$.

Proof. By (1.1),

$$
\begin{aligned}
\Gamma\left(\frac{N-\alpha}{2}\right)\left[2^{\alpha} \pi^{N / 2} \Gamma\left(\frac{\alpha}{2}\right)\right]^{-1}|u-y|^{\alpha-N} \\
\quad=\int_{0}^{\infty} p(t, u-y) d t=(2 \pi)^{-N} \int_{0}^{\infty} d t \int e^{i(\xi, u)} e^{-i(\xi, y)} e^{-t|\xi|^{\alpha}} d \xi .
\end{aligned}
$$

Hence if the bounded signed measure $\nu$ has total variation of finite energy, one may interchange integrals to obtain

$$
\begin{gathered}
\Gamma\left(\frac{N-\alpha}{2}\right)\left[2^{\alpha} \pi^{N / 2} \Gamma\left(\frac{\alpha}{2}\right)\right]^{-1} \int_{A} \nu(d u) \int_{A} \nu(d y)|u-y|^{\alpha-n} \\
=\int_{0}^{\infty} d t \int_{A} \nu(d u) \int_{A} \nu(d y) p(t, u-y) \\
=(2 \pi)^{-N} \int_{0}^{\infty} d t \int|\phi(\xi)|^{2} e^{-t|\xi|^{\alpha}} d \xi
\end{gathered}
$$

where

$$
\phi(\xi)=\int e^{i(\xi, u)} \nu(d u)
$$

is the Fourier-Stieltjes transform of $\nu$. But if

$$
\int_{A}|u-y|^{\alpha-N} \nu(d u)=0, \quad y \in A,
$$

then the energy integral vanishes; hence $\phi=0$, implying $\nu=0$.

Next consider (2.10). Multiplying both sides by

$$
g(y)=\pi^{-1} \sin \frac{\pi \alpha}{2}\left(1-y^{2}\right)^{-\alpha / 2},
$$


and integrating over $|y| \leqq 1$, we have by (3.4) and (3.5)

$$
\begin{aligned}
1-h(x) & =\int_{|y| \leq 1}|x-y|^{\alpha-1} g(y) d y \\
& =\int_{|u| \leq 1} \mu_{x}^{*}(d u) \int_{|y| \leq 1}|u-y|^{\alpha-1} g(y) d y+k_{\alpha}(x) \int_{|y| \leq 1} g(y) d y \\
& =1+\pi^{1 / 2}\left[\Gamma\left(\frac{\alpha}{2}\right) \Gamma\left(\frac{3-\alpha}{2}\right)\right]^{-1} k_{\alpha}(x) .
\end{aligned}
$$

Hence $k_{\alpha}(x)$ is determined from $(2.10)$ by merely the fact that $\mu_{x}^{*}([-1,1])$ $=1$. In particular, if (2.10) has two solutions, each of total mass one, then the difference $\nu$ will satisfy the homogeneous equation

$$
\int_{|u| \leq 1}|u-y|^{\alpha-1} \nu(d u)=0, \quad|y| \leqq 1 .
$$

Moreover, the total variation $|\nu|$ will again have finite energy.

The same remarks apply to (2.6). In fact, multiplying by $g(y)$ $=\pi^{-1}\left(1-y^{2}\right)^{-1 / 2}$ and integrating over $|y| \leqq 1$, by Lemma 3.3

$$
\log \left[|x|+\left(x^{2}-1\right)^{1 / 2}\right]=k_{1}(x), \quad|x|>1,
$$

whenever (2.6) holds for a measure $\mu_{x}^{*}$ of total mass one.

Finally, again in the case of (2.5) and (2.9), the solutions satisfy $\mu_{x}(\{u:|u| \geqq 1\})=1$ and have a finite energy integral. Hence that these four equations determine the hitting probabilities uniquely is a corollary of the following:

Lemma 4.2. Let $G_{\alpha}$ be the kernel defined in $\$ 2$, for $N=1,1 \leqq \alpha<2$. Let $A$ be a closed subset of the real line. Let $\nu$ be a bounded signed measure on $A$ with $\nu(A)=0$, whose total variation $|\nu|$ has finite energy:

$$
\int_{A}|\nu|(d u) \int_{A}|\nu|(d y) G_{\alpha}(u-y)<\infty .
$$

Then

$$
\int_{A} G_{\alpha}(u-y) \nu(d u)=0, \quad y \in A,
$$

implies $\nu=0$.

Proof. Because $|\nu|$ has finite energy,

$$
\int_{A} G_{\alpha}(u-y) \nu(d u)=0, \quad y \in A,
$$

implies 


$$
\begin{aligned}
0 & =\int_{A} \nu(d u) \int_{A} \nu(d y) G_{\alpha}(u-y) \\
& =\lim _{s \rightarrow 0} \int_{A} \nu(d y) \int_{A} \nu(d u)\left[C_{\alpha}(s)-R_{s}(u-y)\right],
\end{aligned}
$$

the integrands $C_{\alpha}(s)-R_{s}(u-y)$ being dominated by $G_{\alpha}(u-y)$. But for $s>0$, since $\nu(A)=0$,

$$
\begin{aligned}
\int_{A} \nu(d y) & \int_{A} \nu(d u)\left[C_{\alpha}(s)-R_{a}(u-y)\right] \\
& =-\int_{A} \nu(d y) \int_{A} \nu(d u) R_{a}(u-y) \\
& =-(2 \pi)^{-1} \int_{A} \nu(d y) \int_{A} \nu(d u) \int e^{i \xi u} e^{-i \xi y}\left(s+|\xi|^{\alpha}\right)^{-1} d \xi \\
= & -(2 \pi)^{-1} \int\left(s+|\xi|^{\alpha}\right)^{-1}|\phi(\xi)|^{2} d \xi,
\end{aligned}
$$

where again $\phi$ is the Fourier-Stieltjes transform of $\nu$. The last integral is bounded away from zero for small $s$, unless $\phi=0$.

5. Concluding remarks. Because of the uniqueness results of the last section, we need only now sort out the various calculations into the proper order.

By (3.7), $f(x ; y)$, for $|x|<1<|y|$, is the unique solution of (2.2). This proves Theorem A for $\alpha<N$, while Theorem B for $\alpha<N$ follows from (3.6) and (2.3). To prove Theorem A for $N=1,1<\alpha<2$, apply (3.7) and (2.9); for $N=1=\alpha$, apply (3.11) and (2.5).

By (3.6) and (3.4), the unique solution of (2.10) is

$$
\mu_{x}^{*}(d y)=f(x ; y) d y-k_{\alpha}(x) g(y) d y,
$$

where by (4.1),

$$
k_{\alpha}(x)=-\pi^{-1 / 2} \Gamma\left(\frac{\alpha}{2}\right) \Gamma\left(\frac{3-\alpha}{2}\right) h(x) .
$$

After a change of variable, this is the result of Theorem $\mathrm{C}$.

Theorem B, when $N=1=\alpha$, follows similarly: (3.9) and (3.10) show that the unique solution of (2.6) is

$$
\begin{aligned}
\mu_{x}^{*}(d y) & =f(x ; y) d y-(\log 2)^{-1}\left\{\log \left[|x|+\left(x^{2}-1\right)^{1 / 2}\right]-k_{1}(x)\right\} g(y) d y \\
& =f(x ; y) d y
\end{aligned}
$$

by (4.2). 
The calculations involved in proving Corollary 1 may be carried out as follows: using again the substitution $y=(1-u x)(x-u)^{-1}$,

$$
\begin{aligned}
\pi \csc \frac{\pi \alpha}{2} p(x) & =\left(1-x^{2}\right)^{\alpha / 2} \int_{1}^{\infty}\left(y^{2}-1\right)^{-\alpha / 2}(y-x)^{-1} d y \\
& =\int_{-1}^{x}(x-u)^{\alpha-1}\left(1-u^{2}\right)^{-\alpha / 2} d u \\
& =(\alpha-1) \int_{-1}^{x} d v \int_{-1}^{v}(v-u)^{\alpha-2}\left(1-u^{2}\right)^{-\alpha / 2} d u \\
& =2^{1-\alpha} \Gamma(\alpha) \Gamma\left(1-\frac{\alpha}{2}\right)\left[\Gamma\left(\frac{\alpha}{2}\right)\right]^{-1} \int_{-1}^{x}\left(1-v^{2}\right)^{\alpha / 2-1} d v
\end{aligned}
$$

if $\alpha>1$. When $\alpha \leqq 1$ the result may be obtained from this by analytic continuation in the parameter $\alpha$.

Corollary 2 is simply a restatement of (3.5): multiplying (3.6) by $g(y)$, as defined in $\S 2$, integrating over $|y| \leqq 1$, and using (3.4) and (3.5),

$$
\begin{aligned}
1-h(x) & =\int_{|y| \leq 1} g(y)|x-y|^{\alpha-N} d y \\
& =\int_{|u| \leq 1} f(x ; u) d u \int_{|y| \leq 1} g(y)|x-y|^{\alpha-N} d y \\
& =\int_{|u| \leq 1} f(x ; u) d u \\
& =1-q(x) .
\end{aligned}
$$

Corollary 3 follows from (2.7) and (4.2), if $\alpha=1$, or from (2.11) and (4.1), if $1<\alpha<2$, upon applying Karamata's Tauberian theorem, since

$$
E_{x}\left[1-e^{-s T^{*}}\right]=s \int_{0}^{\infty} e^{-s t} P_{x}\left[T^{*}>t\right] d t
$$

and since $P_{x}\left[T^{*}>t\right]$ is a decreasing function of $t$ (cf. [11, pp. 208-209]).

Finally, Corollary 4 is immediate from (3.8) and (3.12) applied to (2.4), (2.8) and (2.12).

\section{REFERENCES}

1. R. M. Blumenthal, An extended Markov property, Trans. Amer. Math. Soc. vol. 85 (1957) pp. 52-72.

2. R. K. Getoor, First passage times for symmetric stable processes in space, submitted to Trans. Amer. Math. Soc.

3. M. Kac and $\mathrm{H}$. Pollard, The distribution of the maximum of partial sums of independent random variables, Canad. J. Math. vol. 2 (1950) pp. 375-384. 
4. M. Kac, Some remarks on stable processes, Publ. Inst. Statist. Univ. Paris vol. 6 (1957) pp. 303-306.

5. H. Kesten, Random walks with absorbing barriers and Toeplitz forms, Illinois J. Math., to appear.

6. D. Ray, Stable processes with an absorbing barrier, Trans. Amer. Math. Soc. vol. 89 (1958) pp. 16-24.

7. M. Riesz, Intégrales de Riemann-Liouville et potentiels, Acta. Sci. Math. Szeged. vol. 9 (1938) pp. 1-42.

8. - Rectification au travail "Integrales de Riemann-Liouville et potentiels", Acta Sci. Math. Szeged. vol. 9 (1938) pp. 116-118.

9. F. Spitzer, Some theorems concerning 2-dimensional Brownian motion, Trans. Amer. Math. Soc. vol. 87 (1958) pp. 187-197.

10. H. Widom, Stable processes and integral equations, Trans. Amer. Math. Soc. vol. 98 (1961) pp. 430-449. Cf. also Abstract 571-102, Notices Amer. Math. Soc. vol. 7 (1960) p. 519.

11. G. Doetsch, Theorie und Andwendung der Laplace-Transformation, Berlin, Julius Springer, 1937.

UNIVERSITY OF WASHINGTON,

Seattle, Washington

INSTITUTE FOR ADVANCED STUdY,

Princeton, New Jersey 